\title{
THE ROLE OF ATTENTION IN THE FOOTBALL GAME FOR CHILDREN AND JUNIORS
}

\author{
Gheorghe Dan FETEAN1*, Dan MONEA ${ }^{2}$, Marius ROȘCA ${ }^{3}$ \\ 1 "Nicolae Bălcescu" Theoretical High School, Cluj-Napoca, Romania \\ 2 "Babeș Bolyai" University, Faculty of Physical Education and Sport, Cluj-Napoca, Romania \\ 3 " George Coșbuc" Theoretical High School, Cluj-Napoca, Romania \\ *Corresponding author: feteangheorghe@yahoo.com
}

https://doi.org/10.35189/dpeskj.2021.60.2.11

\begin{abstract}
The influence of attention as a psychological factor on the game of football has a very important role. The difference between an attentive player and a distracted player can destabilise the whole team and can coincide with unwanted results. The current research was born from the desire to require from players something they can achieve, in other words, in vain does the coach ask them to be focused if they have not been taught how to do this or ask them to be vigilant if their attention has never been trained. In order to reach optimal performance, it is primordial for athletes to maintain their attention throughout the game and, if they manage to focus only on the essential information and avoid disrupting factors, they have a good chance to attain peak performance. In this research, we tracked the attentional level of football players, who were initially assessed to determine the exact values, then we intervened with specific exercises for the development of attention, and at the end, we re-assessed attention to see the differences and whether the proposed programme was effective. The research results showed a significant improvement in attentional levels which could increase the sports performance of football players, who managed to progress from pre-test to post-test.
\end{abstract}

Keywords: attention, sports performance, football.

\section{Introduction}

One of the many factors that influence the performance of any sport is attention. In addition, this process has gained crucial importance over the years for achieving sporting success. Athletes who have managed to reach the top of any sport have managed to focus exclusively on the task, avoiding any external distraction. The more carefully the athletes are focused on any task, the better they can develop it and therefore they will get better results. In addition, focused attention is usually assigned to sports jargon, such as "I played the whole match very focused", "I lost my concentration and I couldn't concentrate again", "After the first failure I lost my concentration", affirmations very common for athletes, generally. In short, recurring phrases from the world of sports are a clear sign that concentration is an issue that plays a decisive role in sports competition. Concentration is essential in sports, facilitating the achievement of high sports performance (Hsieh et al., 2010; Grigore et al., 2015; Tüdös et al., 2015), while its deficit in anyone who practices a sport can cost many mistakes. That is why the lack of concentration is considered one of the biggest problems of the sport.

The quality and quantity of athletes' attention is influenced by the fact that there may be cases when the orientation is on other elements, meaning that the individual is distracted by other things around him. We can compare this ability of the athlete to choose where to direct the maximum concentration, with a light bulb always on. For example, during an important 
performance the light bulb is on and the focus must be on the ball, trajectory, etc., but erroneously the performer focuses on the audience or is influenced by other disruptive factors. To try to alleviate the harmful effects of lack of concentration in sports, coaches use a number of strategies. Although motivation and activation can help athletes in order to maintaining adequate concentration, sports professionals have found that the presence of sports psychologists can help avoid mistakes due to lack of concentration.

Psychological training is particularly important in a coach's activity because it represents one of the key points for achieving remarkable results. An analogy was made between the levels of stress in basketball players and football players, which demonstrated that, during a competition, players who had benefited from specific training showed slightly moderate stress compared to the other participants (Kollos \& Tache, 2013).

The technical steps to conduct psychological training can be applied by coaches over short stages, but long-term qualified psychological training must be provided by psychologists. Psychology seeks to examine and understand the causal process that contributes to behaviour change. "It attempts to discover why organisms behave differently by understanding, analyzing, predicting and controlling behavior of organisms. It is therefore that branch of science which is concerned with the behavior of organisms." (Ohuruogu et al., 2016, p. 47)

Sport psychology places great emphasis on the importance of understanding both the behaviour of individuals engaged in sports activity and the characteristics of sports groups. A football team is a special type of group. In addition to bilateral influences and common tasks, teams are distinguished by four specific characteristics:

- a sense of collective identity;

- distinct roles: each member knows well their responsibilities;

- structured ways of communication;

- social norms and rules that guide the group members in their activity. (Weinberg \& Gould, 2007)

Howe (1991), who developed the model of training general mental ability, structured psychological preparation into two categories:

- First-order components - pre-competitive and competitive strategies, confidence building and concentration;

- Second-order components - performance goal-setting, attentional strategies, communication skills, relaxation ability, etc.

In team sports such as football, attention is not focused only on physical ability and coordination but cognitive abilities are also very important, which indicates that executive functions play a crucial role in the game of football. Simple executive functions, those related to memory ability, have a faster pace of development than complex executive functions, which represent the manipulation of information. Vestberg et al. (2017) have demonstrated in their study that executive functions can predict football success in both children and juniors.

Researchers have studied attentional orienting in sport, and some of their findings show that:

- participants in open-skill sports such as boxing (Nougier et al., 1989), hockey (Enns \& Richards, 1997), pentathlon (Nougier et al., 1989), football (Lum et al., 2002) or volleyball (Castiello \& Umiltà, 1992) have a higher level of visual attentional orienting compared to 
novices. Athletes may prefer to pay less attention to high probability events and more attention to less likely events. This is probably not the case of athletes who are engaged in closed-skill sports (Nougier, 1996);

- professionals, more precisely experienced volleyball players (Pesce Anzeneder \& Bosel, 1998) and skiers, can modulate their attention according to task-specific requirements (Castiello \& Umiltà, 1992). Turatto et al. (1999) have highlighted that the attentional focus dimension is more efficiently adapted in professionals, which means that, depending on the task, they are able to better modulate their attentional focus dimension compared to novices. Exercise or submaximal load leads to reduced reaction time among professional athletes and, especially for invalid cues, attention reaction costs have decreased in terms of time (Pesce et al., 2004);

- attentional orienting can also have an influence on the control of a motor ability during its execution or the performance of a decision-making task specific to each sport (Lépine et al., 1989).

First of all, research on the "flow states" of athletes and experiences of maximum sports performance highlight the importance of being totally absorbed into the task for optimal performance. Second, there is growing evidence of a connection between the attentional orienting of athletes and the quality of their performance (Harmison, 2007). Attention stability consists in the long-term maintenance of attentional focus on the same object or activity. Stability does not mean "freezing" attention in a state of fascination but involves certain fluctuations that, however, do not interrupt the main focus of the activity.

Due to attentional fluctuations, variations in the efficiency of a long-term activity are possible to occur. Decreases in efficiency are all the more pronounced as the activity requires a higher focus of attention. Fluctuations in attention occur as an effect of protective inhibition. If they do not change the main focus of the activity, they represent adaptive phenomena that ensure the moments of rest necessary during a long-term activity.

The stability of attention is easier to achieve in the case of an action with moving objects or that involves certain changes. This quality is particularly important in areas of activity that require the instantaneous observation of as many elements as possible.

The flexibility of attention consists in the ability to shift attention from one object to another within short intervals and is influenced by the specifics of the activities carried out. Thus, the attentional shift is more difficult to achieve when an activity has not ended, and the new activity is less interesting and much different from the previous one. Flexibility should not be confused with involuntary changes, which represent distracting phenomena.

The distribution of attention is characterised by the number of activities that a person can perform simultaneously while none interferes too much with the others. Simultaneity is achieved by rapidly shifting attention from one activity to another, by including actions into a single coordinated activity or by virtue of the fact that one of the activities is automated and does not require attentional control.

Concentration is probably the most important dimension of attention, which expresses the degree of selective activation and the intensity of the dominant foci at the level of brain structures and areas involved in carrying out the specific psychological process or activity. It can thus take different values both from one person to another and for one and the same 
person at different times, depending on the characteristics and content of the tasks but also on its internal state (relating to motivation, emotions, rest-fatigue, etc.).

Like the other dimensions of attention, distributivity can be best educated and shaped within the profession. There are professions such as the executive-motor, driving, leading or teaching ones, which require full distributivity or at least fast switching between several different actions or links.

Wulf (2013) has concluded that an external focus is usually more effective than an internal focus in enhancing the learning and performance of various motor skills. This finding seems to generally apply to a number of tasks (e.g., balance and jumping activities, golf, football) and levels of experience (i.e., whether athletes are professionals or novices).

Finally, a variety of anecdotal testimonies and sports incidents emphasise the importance of concentration skills in determining athletic performance. To illustrate, Petr Cech, the Chelsea goalkeeper who kept a clean sheet in 23 Premiership Matches in 2005, revealed that "it's all about concentration". This comment was determined by Cech's observation that opposing teams invariably have at least one chance to score during a game, and therefore it is difficult to be focused for the right moment. Clearly, decreased attention can make the difference between success and failure in sports activities (Szczepanik, 2005).

It is noticed that attention is not homogeneous and unidimensional but a complex picture that is divided into three main parts as follows: involuntary attention, voluntary attention and post-voluntary attention (Gould et al., 2002).

\section{Research purpose}

The current paper aims to study the role of attention in the game of football at the age of 9-10 years; more specifically, its purpose is to demonstrate the efficiency of an intervention program in the development of attention.

\section{Methodology}

\section{Participants}

The participants in this research were 30 male athletes (15 in the experimental group and 15 in the control group) aged 10 years, members of the ACS BEST JUNIOR Football Club, Romania.

\section{Instruments}

Two assessment instruments were used in this study, namely the EM-05.48 C Focused Attention Tester and the Combined EM-05.54, EM-05.55 Distributed Attention Tester.

The EM-05.48 C Focused Attention Tester is controlled by microprocessors and provides modern electronic solutions to assess focused attention, attentional stability and fluctuations, the rapidity of observing and understanding information, dysfunctions in understanding working tasks, risk-taking willingness. It "can be used in all fields where sustained and continuous attention plays an important role in activity" (Fetean et al., p. 42). Examination 
performed with this tester can be included in various studies "because measurements also provide information about memory qualities" and "the degree of resistance to monotony" (Fetean et al., p. 43).

The Combined EM-05.54, EM-05.55 Distributed Attention Tester assesses distributed attention and hand-eye coordination. It can be successfully used for skill testing in activities that require a high level of attention as well as a testing tool in educational counselling. Thus, it is useful in the fields of both psychological skill testing and scientific research. There is a connection between attention and vigilance, which is why the obtained results represent an important milestone in the assessment of player's capability.

With the help of these two devices, tests will be performed to determine the attentional level of players. The participants in this research will perform, in addition to the footballspecific training, exercises for the development of attention as a psychological factor.

\section{Procedure}

The research was conducted over a 6-month period during which the intervention programme was applied in order to see whether the proposed means helped to increase focused attention and distributed attention. The research started on January 4, 2019, and the intervention programme lasted until July 1, 2019.

The final test was performed between June 25-30, under the same conditions and using the same tests as for the initial test. The number of planned workouts was 3 per week (every Monday, Wednesday and Friday), and each one lasted 1 hour and 20 minutes (in the case of the experimental group), from 16:00 to 17:20. In addition to the football training programme, the proposed intervention programme was applied to the experimental group at the beginning of each session for 20 minutes. No intervention programme was used for the control group, who followed the specific schedule, namely 3 workouts per week of 1 hour each.

In order to develop focused attention and distributed attention, a number of age-specific football games were used.

- Methods to develop focused attention

Players are arranged in a row, with a ball at each one's foot. Each player performs 3 shots from 8-10 m towards a fixed point: a red card is attached to the goal net, which players have to hit in turn. Each athlete needs to be given time to focus without being disturbed by other teammates. The exercise can be performed simultaneously at the two goals, with players divided into two equal groups.

- Methods to develop distributed attention

Players are placed on the midfield line, 4 or 5 of them lying down with palm support. Those with the ball have to dribble the players lying down and reach the end line. The latter try to touch the others' balls. Players who lose their balls become "crabs". The game is very efficient for the development of technique and distributed attention. 


\section{Results}

In an initial stage we checked if there are significant differences between the two groups of participants before the experimental intervention. Since $p>0.05$ we specify that in terms of concentrated and distributive attention, there are no significant differences between the experimental group and the control group at the beginning of the study. For an objective appreciation, we present the mean differences between the experimental group and the control group in the initial phase of the research (before the experimental intervention) in the case of attention: mean difference $=-0.15$ (Correct reactions - Distributed attention); mean difference $=0.70$ (Erroneous reactions - Distributed attention); mean difference $=-0.20$ (Average performance - Distributed attention); mean difference $=0.34$ (Dispersion Distributed attention); mean difference $=-1.68$ (Average percentage of errors per minute Distributed attention); mean difference $=1.05$ (Correct responses - Focused attention); mean difference $=-0.13$ (Erroneous responses - Focused attention); mean difference $=-0.10$ (Average reaction time - Focused attention); mean difference $=-0.43$ (Dispersion - Focused attention).

We further present the results of the athletes at the final testing in the case of concentrated and distributive attention.

The average number of correct reactions in the case of distributed attention is lower by $0.35(0.27 \%)$ for the experimental group. The means have the values 131.18 for the experimental group and 131.53 for the control group. The correct reactions are homogeneously spread for both groups. The mean difference between groups is very small. The Mann-Whitney test shows a statistically insignificant difference, the significance threshold $\mathrm{p}=0.925>0.05$ for $\mathrm{Z}=-0.094$ (Table 1 ).

Table 1. Correct reactions - Distributed attention

\begin{tabular}{lcc}
\hline \multicolumn{1}{c}{ Statistical indicators } & Experiment & Control \\
\hline Mean & 131.18 & 131.53 \\
Median & 137.00 & 139.00 \\
Standard deviation & 15.42 & 17.22 \\
Minimum & 97.0 & 103.0 \\
Maximum & 155.0 & 160.0 \\
Range & 58.0 & 57.0 \\
Coefficient of variation & $11.8 \%$ & $13.1 \%$ \\
\hline
\end{tabular}

\begin{tabular}{lcc}
\hline \multicolumn{2}{c}{ Mean difference (E-C) } \\
\hline Mean difference & -0.35 \\
Mean difference (\%) & $0.27 \%$ \\
\hline \multicolumn{2}{c}{ Significance test } \\
\multicolumn{2}{c}{ Z } \\
\hline $\begin{array}{lc}\text { Non-parametric Mann- } \\
\text { Whitney test }\end{array}$ & -0.094 & 0.925 \\
\hline Effect size & 0.02 \\
\hline
\end{tabular}

For distributed attention, the average number of erroneous reactions is lower by 1.53 correct reactions (44.0\%) for the experimental group. The means have the values 1.94 for the experimental group and 3.47 correct reactions for the control group. The obtained results have an inhomogeneous structure for both groups. The mean difference between groups is medium to large. The Mann-Whitney test shows that this difference is statistically significant, the significance threshold $\mathrm{p}=0.009<0.05$ for $\mathrm{Z}=-2.620$ (Table 2 ). 
Table 2. Erroneous reactions - Distributed attention

\begin{tabular}{lcc}
\hline \multicolumn{1}{c}{ Statistical indicators } & Experiment & Control \\
\hline Mean & 1.94 & 3.47 \\
Median & 2.00 & 3.00 \\
Standard deviation & 1.03 & 1.85 \\
Minimum & 0.0 & 0.0 \\
Maximum & 4.0 & 7.0 \\
Range & 4.0 & 7.0 \\
Coefficient of variation & $53.0 \%$ & $53.3 \%$ \\
\hline
\end{tabular}

\begin{tabular}{lcc}
\hline \multicolumn{2}{c}{ Mean difference (E - C) } \\
\hline Mean difference & -1.53 \\
Mean difference (\%) & $44.0 \%$ \\
\hline \multicolumn{2}{c}{ Significance test } \\
\multicolumn{2}{c}{$\mathrm{Z}$} \\
\hline Non-parametric Mann- \\
\cline { 2 - 3 } Whitney test & -2.620 & 0.009 \\
\hline Effect size & 0.46 \\
\hline
\end{tabular}

The average performance in the case of distributed attention is lower by $0.15(0.57 \%)$ for the experimental group. The means have the values 26.24 for the experimental group and 26.39 for the control group. The obtained results show a homogeneous structure for both groups. The mean difference between the groups is very small. The Mann-Whitney test indicates that this difference is statistically insignificant, the significance threshold $p=0.925$ $>0.05$ for $\mathrm{Z}=-0.094$ (Table 3 ).

Table 3. Average performance - Distributed attention

\begin{tabular}{lcc}
\hline \multicolumn{1}{c}{ Statistical indicators } & Experiment & Control \\
\hline Mean & 26.24 & 26.39 \\
Median & 27.40 & 27.80 \\
Standard deviation & 3.08 & 3.60 \\
Minimum & 19.4 & 20.6 \\
Maximum & 31.0 & 33.2 \\
Range & 11.6 & 12.6 \\
Coefficient of variation & $11.8 \%$ & $13.6 \%$ \\
\hline
\end{tabular}

\begin{tabular}{lcc}
\hline \multicolumn{2}{c}{ Mean difference (E-C) } \\
\hline Mean difference & -0.15 \\
Mean difference (\%) & $0.57 \%$ \\
\hline \multicolumn{2}{c}{ Significance test } \\
\multicolumn{2}{c}{ Z } \\
\hline Non-parametric Mann- \\
Whitney test & -0.094 & 0.925 \\
\hline Effect size & 0.02 \\
\hline
\end{tabular}

The average dispersion in the case of distributed attention is lower by $0.75(24.8 \%)$ for the experimental group. The means are 2.26 for the experimental group and 3.01 for the control group. The distribution of results is inhomogeneous for both groups. The mean difference between groups is small to medium. The Mann-Whitney test shows that this difference is statistically insignificant, the significance threshold $\mathrm{p}=0.135>0.05$ for $\mathrm{Z}=-1.496$ (Table 4).

Table 4. Dispersion - Distributed attention

\begin{tabular}{lcc}
\hline \multicolumn{1}{c}{ Statistical indicators } & Experiment & Control \\
\hline Mean & 2.26 & 3.01 \\
Median & 1.90 & 2.90 \\
Standard deviation & 1.19 & 1.52 \\
Minimum & 0.7 & 0.7 \\
Maximum & 5.4 & 6.0 \\
Range & 4.7 & 5.3 \\
Coefficient of variation & $52.5 \%$ & $50.4 \%$ \\
\hline
\end{tabular}

\begin{tabular}{lcc}
\hline \multicolumn{2}{c}{ Mean difference (E-C) } \\
\hline Mean difference & -0.75 \\
Mean difference (\%) & $24.8 \%$ \\
\hline \multicolumn{2}{c}{ Significance test } \\
\multicolumn{2}{c}{$\mathrm{Z}$} & $\mathrm{P}$ \\
\hline Non-parametric Mann- & -1.496 & 0.135 \\
Whitney test & & 0.26 \\
\hline Effect size
\end{tabular}

The average percentage of errors per minute in the case of distributed attention is higher by $1.1(1.1 \%)$ for the experimental group. The means have the values $98.5 \%$ for the experimental group and $97.4 \%$ for the control group. The data are homogeneously spread for both groups. The mean difference between groups is medium to large. The Mann-Whitney 
test shows that this difference is statistically significant, the significance threshold $\mathrm{p}=0.013$ $<0.05$ for $\mathrm{Z}=-2.480$ (Table 5).

Table 5. Average percentage of errors per minute - Distributed attention

\begin{tabular}{lcc}
\hline \multicolumn{1}{c}{ Statistical indicators } & Experiment & Control \\
\hline Mean & $98.5 \%$ & $97.4 \%$ \\
Median & $98.6 \%$ & $97.5 \%$ \\
Standard deviation & $0.8 \%$ & $1.3 \%$ \\
Minimum & $97.3 \%$ & $95.7 \%$ \\
Maximum & $100.0 \%$ & $100.0 \%$ \\
Range & $2.7 \%$ & $4.3 \%$ \\
Coefficient of variation & $0.8 \%$ & $1.3 \%$ \\
\hline
\end{tabular}

\begin{tabular}{lcc}
\hline \multicolumn{2}{c}{ Mean difference (E-C) } \\
\hline Mean difference & $1.1 \%$ \\
Mean difference (\%) & $1.1 \%$ \\
\hline \multicolumn{2}{c}{ Significance test } \\
\multicolumn{2}{c}{ Zon-parametric Mann- } & $\mathrm{P}$ \\
\cline { 2 - 3 } Whitney test & -2.480 & 0.013 \\
\hline Effect size & 0.44 \\
\hline
\end{tabular}

The average number of correct responses in the case of focused attention is higher by 3.32 (13.1\%) for the experimental group. The means are 28.65 for the experimental group and 25.33 for the control group. The obtained results show a relatively homogeneous structure for both groups. The mean difference between the two groups is small to medium. The MannWhitney test indicates that this difference is statistically insignificant, the significance threshold $\mathrm{p}=0.098>0.05$ for $\mathrm{Z}=-1.657$ (Table 6).

Table 6. Correct responses - Focused attention

\begin{tabular}{lcc}
\hline \multicolumn{1}{c}{ Statistical indicators } & Experiment & Control \\
\hline Mean & 28.65 & 25.33 \\
Median & 29.00 & 26.00 \\
Standard deviation & 5.30 & 7.03 \\
Minimum & 20.0 & 14.0 \\
Maximum & 39.0 & 39.0 \\
Range & 19.0 & 25.0 \\
Coefficient of variation & $18.5 \%$ & $27.7 \%$ \\
\hline
\end{tabular}

\begin{tabular}{lcc}
\hline \multicolumn{2}{c}{ Mean difference (E-C) } \\
\hline Mean difference & 3.32 \\
Mean difference (\%) & $13.1 \%$ \\
\hline \multicolumn{2}{c}{ Significance test } \\
\multicolumn{2}{c}{ Non-parametric Mann- } & $\mathrm{P}$ \\
\cline { 2 - 3 } Whitney test & -1.657 & 0.098 \\
\hline Effect size & 0.29 \\
\hline
\end{tabular}

For focused attention, the average number of erroneous responses is lower by 0.56 (44.3\%) for the experimental group. The means have the values 0.71 for the experimental group and 1.27 for the control group. The distribution of results is inhomogeneous for both groups. The mean difference between the two groups is small to medium. The Mann-Whitney test shows that this difference is statistically insignificant, the significance threshold $\mathrm{p}=$ $0.332>0.05$ for $\mathrm{Z}=-0.971$ (Table 7).

Table 7. Erroneous responses - Focused attention

\begin{tabular}{lcc}
\hline \multicolumn{1}{c}{ Statistical indicators } & Experiment & Control \\
\hline Mean & 0.71 & 1.27 \\
Median & 0.00 & 1.00 \\
Standard deviation & 0.92 & 1.62 \\
Minimum & 0.0 & 0.0 \\
Maximum & 3.0 & 6.0 \\
Range & 3.0 & 6.0 \\
Coefficient of variation & $130.3 \%$ & $128.2 \%$ \\
\hline
\end{tabular}

\begin{tabular}{lcc}
\hline \multicolumn{2}{c}{ Mean difference (E-C) } \\
\hline Mean difference & -0.56 \\
Mean difference (\%) & $44.3 \%$ \\
\hline \multicolumn{2}{c}{ Significance test } \\
\multicolumn{2}{c}{$\mathrm{Z}$} & $\mathrm{P}$ \\
\hline Non-parametric Mann- & -0.971 & 0.332 \\
Whitney test & & 0.17 \\
\hline Effect size & \\
\hline
\end{tabular}


The average reaction time obtained by athletes in the case of focused attention is lower by $0.62(4.3 \%)$ for the experimental group. The means have the values 13.69 for the experimental group and 14.31 for the control group. The obtained results show a relatively homogeneous structure for both groups. The mean difference between groups is very small. The Mann-Whitney test indicates that this difference is statistically insignificant, the significance threshold $\mathrm{p}=0.719>0.05$ for $\mathrm{Z}=-0.359$ (Table 8).

Table 8. Average reaction time - Focused attention

\begin{tabular}{lcc}
\hline \multicolumn{1}{c}{ Statistical indicators } & Experiment & Control \\
\hline Mean & 13.69 & 14.31 \\
Median & 13.40 & 13.40 \\
Standard deviation & 2.60 & 3.85 \\
Minimum & 7.8 & 7.8 \\
Maximum & 19.8 & 21.8 \\
Range & 12.0 & 14.0 \\
Coefficient of variation & $19.0 \%$ & $26.9 \%$ \\
\hline
\end{tabular}

\begin{tabular}{lcc}
\hline \multicolumn{2}{c}{ Mean difference (E-C) } \\
\hline Mean difference & -0.62 \\
Mean difference (\%) & $4.3 \%$ \\
\hline \multicolumn{2}{c}{ Significance test } \\
\multicolumn{2}{c}{ Z } \\
\hline Non-parametric Mann- \\
Whitney test & -0.359 & 0.719 \\
\hline Effect size & 0.06 \\
\hline
\end{tabular}

The mean dispersion recorded in the case of focused attention is lower by 1.34 (7.1\%) for the experimental group. The means have the values 17.58 for the experimental group and 18.92 for the control group. The dispersion of results is inhomogeneous for both groups. The mean difference between groups is very small. The Mann-Whitney test shows that this difference is statistically insignificant, the significance threshold $\mathrm{p}=0.762>0.05$ for $\mathrm{Z}=-$ 0.303 (Table 9).

Table 9. Dispersion - Focused attention

\begin{tabular}{lcc}
\hline \multicolumn{1}{c}{ Statistical indicators } & Experiment & Control \\
\hline Mean & 17.58 & 18.92 \\
Median & 18.40 & 18.80 \\
Standard deviation & 5.55 & 8.23 \\
Minimum & 8.0 & 8.0 \\
Maximum & 27.1 & 42.5 \\
Range & 19.1 & 34.5 \\
Coefficient of variation & $31.6 \%$ & $43.5 \%$ \\
\hline
\end{tabular}

\begin{tabular}{lcc}
\hline \multicolumn{2}{c}{ Mean difference (E-C) } \\
\hline Mean difference & -1.34 \\
Mean difference (\%) & $7.1 \%$ \\
\hline \multicolumn{2}{c}{ Significance test } \\
\multicolumn{2}{c}{$\mathrm{Z}$} \\
\hline Non-parametric Mann- \\
\cline { 2 - 3 } Whitney test & -0.303 & 0.762 \\
\hline Effect size & & 0.05 \\
\hline
\end{tabular}

\section{Conclusion}

The theoretical originality of this paper consists in the fact that it highlights a new trend in the training process as a result of implementing the psychological training programme, with possible implications in increasing the sports performance of football players.

According to the theoretical research already existing in the literature, the starting point was the idea that the implementation of an intervention programme in football training would increase the attentional level of football players aged 9-10 years. During the research, an initial test and a final test were conducted to assess attention as a psychological factor with its two forms, distributed attention and focused attention, through various specialised tests. For all tests, participants from the experimental group show better performances (after applying the proposed programme), comparing to the control group. 
The athletes who improved their attentional level after the intervention achieved significantly better results compared to the control group in the case of the average number of erroneous reactions and for the average percentage of errors per minute in the case of distributed attention. The players' performance was influenced by the on-court psychological training programme, their attentional level increasing, which could help them better understand the exercises in technical terms and the tactical requirements transmitted by the coach. An increased level of attention could positively influence sports performance for football players aged 9-10 years.

The current scientific research provides more information considering focused and distributed attention in young football players. As regards the sport psychology studies focused on younger football players, the information is less approached. Besides the fact that this paper brings a new source in the literature, it also draws attention that this area needs further research.

\section{References}

Castiello, U., \& Umiltà, C. (1992). Orienting of attention in volleyball players. International Journal of Sport Psychology, 23(4), 301-310.

Enns, J. T., \& Richards, J. C. (1997). Visual attentional orienting in developing hockey players. Journal of Experimental Child Psychology, 64(2), 255-275. https://doi.org/10.1006/jecp.1996.2348

Fetean, Gh. D., Monea, Gh., \& Roșca, M. (2020). The influence of attention in increasing sports performance in football at the age of 7-10 years. Health, Sports and Rehabilitation Medicine, 21(1), 41-44.

Gould, D., Dieffembach, K., \& Moftet, A. (2002). Psychological characteristics and their development in Olympic champions. Journal of Applied Sport Psychology, 14(3), 172204. https://doi.org/10.1080/10413200290103482

Grigore, V., Predoiu, R., \& Păunescu, P. (2015). The ability to focus attention - predictor for the decision time in sport. Proceedings of the 11th International Scientific Conference "eLearning and Software for Education", 3, 254-259. DOI: 10.12753/2066-026X-15-219

Harmison, R. J. (2007). Peak performance in sport: Identifying ideal performance states and developing athletes' psychological skills. Professional Psychology: Research and Practice, 37(3), 233-243. http://dx.doi.org/10.1037/0735-7028.37.3.233

Howe, B. L. (1991). Imagery and sport performance. Sports Medicine, 11(1), 1-5. https://doi.org/10.2165/00007256-199111010-00001

Hsieh, T., Huang, C., \& Hung, T., 2010. Relationships between heart rate variability, attention, and athletic performance. International Journal of Sport and Exercise Psychology, 8(4), 473-475. https://doi.org/10.1080/1612197X.2010.9671964

Kollos, C., \& Tache, S. (2013). Psychological stress in junior basketball players. Palestrica of the Third Millennium - Civilization and Sport, 14(2), 118-122.

Lépine, D., Glencross, D., \& Requin, J. (1989). Some experimental evidence for and against a parametric conception of movement programming. Journal of Experimental Psychology: Human Perception and Performance, 15(2), 347-362. https://doi.apa.org/doi/10.1037/0096-1523.15.2.347

Lum, J., Enns, J. T., \& Pratt, J. (2002). Visual orienting in the college athletes: Explorations of athlete type and gender. Research Quarterly for Exercise and Sport, 73(2), 156-167. https://doi.org/10.1080/02701367.2002.10609004 
Nougier, V., Ripoll, H., \& Stein, J.-F. (1989). Orienting of attention with highly skilled athletes. International Journal of Sport Psychology, 20(3), 205-223.

Nougier, V., Rossi, B., Alain, C., \& Taddei, F. (1996). Evidence of strategic effects in the modulation of orienting of attention. Ergonomics, 39(9), 1119-1133. https://doi.org/10.1080/00140139608964533

Ohuruogu, B., Jonathan, U. I., \& Ikechukwu, U. J. (2016). Psychological preparation for peak performance in sport competition. Journal of Education and Practice, 7(12), 47-50.

Pesce, C., Casella, R., \& Capranica, L. (2004). Modulation of visuospatial attention at rest and during physical exercise: Gender differences. International Journal of Sport Psychology, 35(4), 328-341.

Pesce Anzeneder, C., \& Bosel, R. (1998). Modulation of the spatial extent of the attentional focus in high-level volleyball players. European Journal of Cognitive Psychology, 10(3), 247-267. https://doi.org/10.1080/713752275

Szczepanik, N. (2005, April 30). Focused Cech puts records low on his list of priorities. The Times. https://www.thetimes.co.uk/article/focused-cech-puts-records-low-on-his-list-ofpriorities-nqnrdbgkkdb

Turatto, M., Benso, F., \& Umiltà, C. (1999). Focusing of attention in professional women skiers. International Journal of Sport Psychology, 30, 339-349.

Tüdös, S., Predoiu, R., \& Predoiu, A. (2015). Topographical memory and the concentration of attention in top female tennis players. Procedia - Social and Behavioral Sciences, 190, 293-298. https://doi.org/10.1016/j.sbspro.2015.05.001

Vestberg, T., Reinebo, G., Maurex, L., Ingvar, M., \& Petrovic, M. (2017). Core executive functions are associated with success in young elite soccer player. PLOS ONE, 1-13. https://doi.org/10.1371/journal.pone.0170845

Weinberg, R. S., \& Gould, D. (2007). Foundations of sport and exercise psychology (4th ed.). Human Kinetics.

Wulf, G. (2013). Attentional focus and motor learning: A review of 15 years. International Review of Sport and Exercise Psychology, 6(1), 77-104. http://dx.doi.org/10.1080/1750984X.2012.723728 